\title{
Azathioprine-induced severe anemia potentiated by the concurrent use of allopurinol
}

\author{
Lorenzo Madrazo MD, Emily Jones MD, Cyrus C. Hsia MD
}

Cite as: CMAJ 2021 January 18;193:E94-7. doi: 10.1503/cmaj.201022

A 66-year-old man presented to the emergency department with a 2-week history of progressive weakness and lethargy. Three months before presentation, he had been started on azathioprine therapy for immunoglobulin (Ig) G4-related biliary disease. Comorbidities included hypertension, peripheral vascular disease, type 2 diabetes mellitus, salivary gland fibrosis, hypothyroidism, gastresophageal reflux disease, hyperlipidemia, osteoarthritis and gout. The patient was taking azathioprine $200 \mathrm{mg}$ once daily and had been taking allopurinol $100 \mathrm{mg}$ once daily for several years to manage his gout. Other medications included sitagliptin $100 \mathrm{mg}$ once daily, gliclazide $120 \mathrm{mg}$ once daily, acetylsalicylic acid $81 \mathrm{mg}$ once daily, extended-release metoprolol $200 \mathrm{mg}$ once daily, ramipril $5 \mathrm{mg}$ once daily, atorvastatin $40 \mathrm{mg}$ once daily, rabeprazole $20 \mathrm{mg}$ twice daily, clonazepam $2 \mathrm{mg}$ at bedtime, gabapentin $100 \mathrm{mg}$ 3 times daily, venlafaxine $225 \mathrm{mg}$ daily, vitamin D 1000 IU once daily and ibuprofen $800 \mathrm{mg}$ as needed.

On examination, the patient was hypotensive at 92/45 $\mathrm{mm} \mathrm{Hg}$. He appeared mildly fatigued and was not pale or jaundiced. There was no hepatosplenomegaly or abdominal mass, and a digital rectal exam did not show any melena.

Initial laboratory investigations showed that the patient had a hemoglobin of 56 (normal range 135-170) g/L which was down from $86 \mathrm{~g} / \mathrm{L}$ and $158 \mathrm{~g} / \mathrm{L}, 1$ month and 3 months before, respectively. His other blood test results were as follows: mean corpuscular volume 107.8 (normal range 80.0-100.0) fL $(90.0 \mathrm{fL}$ 3 months before), white blood cell count 3.2 (normal range 4.0$10.0) \times 10^{9} / \mathrm{L}$, neutrophils 2.1 (normal range $2.0-7.5$ ) $\times 10^{9} / \mathrm{L}$, lymphocytes 0.6 (normal range $1.0-4.0$ ) $\times 10^{9} / \mathrm{L}$ and platelet count of 194 (normal range $150-400) \times 10^{9} / \mathrm{L}$. The patient received a blood transfusion in the emergency department and his blood pressure improved to $127 / 70 \mathrm{~mm} \mathrm{Hg}$. Our differential diagnosis included an active bleed from an intra-abdominal source, hemolysis, malignant disease and drug-induced anemia.

On admission to hospital, azathioprine was stopped because of its known myelosuppressive effect. Acetylsalicylic acid, ramipril and metoprolol were also stopped on admission because of concerns related to bleeding and hypotension. A reticulocyte count measured the day after his admission was 71 (normal range 10-100) × 10\%/L.

\section{KEY POINTS}

- Severe anemia and myelosuppression are rare but serious complications of azathioprine that are more likely to occur at high doses or when potentiated by interactions with other drugs.

- Xanthine oxidase inhibitors such as allopurinol or febuxostat increase the production of myelotoxic metabolites from azathioprine.

- Initiation of azathioprine should be accompanied by regular monitoring of a complete blood count with differential and liver enzymes at least every 2 weeks during initial dose titration, and, once stable, at least every 3 months thereafter, as clinically appropriate.

Investigations for anemia included upper and lower endoscopy that showed no gastrointestinal source of bleeding. A computed tomography scan of the abdomen and pelvis did not suggest intra-abdominal or retroperitoneal bleeding. Investigations for possible hemolysis included the following: lactate dehydrogenase 339 (normal range < 225) U/L, total bilirubin 15.8 (normal 3.4-17.1) $\mu \mathrm{mol} / \mathrm{L}$, direct bilirubin 6.8 (normal 0.0-5.0) $\mu \mathrm{mol} / \mathrm{L}$ and haptoglobin 0.79 (normal 0.3-2.0) g/L. A direct antiglobulin test was negative for IgG and complement. The patient's serum vita$\min B_{12}$ level, thyroid stimulating hormone and iron profile were all within normal limits. A bone marrow biopsy showed normocellular trilineage hematopoietic marrow with megaloblastoid features, but no convincing morphological features that met the criteria for myelodysplasia. Cytogenetic studies on the bone marrow aspirate were normal.

Having ruled out a source of active bleeding as well as hemolytic and malignant processes, we focused on a diagnosis of druginduced anemia related to azathioprine. We consulted the clinical pharmacology team, who tested for thiopurine methyltransferase (TPMT) gene mutations. They also suggested stopping the patient's allopurinol, because anemia has been linked to an interaction between allupurinol and azathioprine. Angiotensinconverting-enzyme inhibitors such as ramipril - which the patient had been taking - have also been associated with anemia and 
leukopenia when taken concurrently with azathioprine. ${ }^{1}$ However, this association has exclusively been reported in patients who underwent renal transplantation; in this context, anemia is thought to be related to decreased erythropoietin levels. ${ }^{1}$ Our patient's test results for TPMT mutations were negative; therefore, we concluded that his anemia was most likely related to azathioprine use, potentiated by allopurinol. He received a total of 3 units of packed red blood cells and his hemoglobin remained stable throughout admission. His leukocyte counts also recovered gradually. After 1 week in hospital, we discharged our patient.

A follow-up visit 2 weeks after stopping azathioprine and allopurinol showed that our patient's hemoglobin had continued to rise, from $78 \mathrm{~g} / \mathrm{L}$ at the time of hospital discharge to $100 \mathrm{~g} / \mathrm{L}$. Repeated blood tests over the next 3 months showed continued improvement in hemoglobin levels (Figure 1). The patient opted to remain off azathioprine therapy and we prescribed mycophenolate sodium $720 \mathrm{mg}$ twice daily as an alternative agent for his IgG4-related biliary disease. He also remained off allopurinol and has not had any recurrent flares of gout.

Using the World Health Organization Uppsala Monitoring Centre causality categories, it is "probable" that azathioprine was responsible for our patient's severe anemia. ${ }^{2}$ This is based on the temporal relation between initiation and onset of laboratory abnormalities, the low likelihood of other causes and the patient's response to stopping azathioprine. ${ }^{2}$ As per the patient's wishes, he was not rechallenged with azathioprine. We reported this case to the Canada Vigilance Program, a postmarket surveillance program by Health Canada that collects reports of suspected adverse reactions to health products.

\section{Discussion}

\section{Azathioprine and myelosuppression}

Azathioprine is a commonly used immunosuppressant, and is approved by Health Canada for the treatment of rheumatoid arthritis and for the prevention of graft rejection in renal transplant patients. ${ }^{3}$ It also has several off-label uses for inflammatory bowel disease, IgG4-related biliary disease and other transplant populations such as heart, lung and liver. ${ }^{3-5}$ Myelosuppression as evidenced by leukopenia ${ }^{5-7}$ is a common adverse effect and is attributed to high drug metabolite levels in a dose-dependent manner. ${ }^{6}$

Severe anemia is an uncommon manifestation of azathioprinerelated myelosuppression, and has been described in case reports as megaloblastic anemia or pure red cell aplasia. ${ }^{8}$ In our patient, myelosuppression manifested as both leukopenia and severe anemia.

\section{Azathioprine pharmacology and interaction with other drugs}

Azathioprine, a prodrug, is metabolized into its active form, 6-mercaptopurine, by a nonenzymatic process. As Figure 2 shows, 6-mercaptopurine is subsequently metabolized through 3 pathways: by TPMT into 6-methylmercaptopurine, by xanthine oxidase into 6-thiouracil, and by hypoxanthine guanine phosphoribosyltransferase into 6-thioguanine. ${ }^{6}$ Inhibition of the TPMT or xanthine oxidase pathways leads to increased 6-mercaptopurine bioavailability, which in turn increases the levels of the toxic metabolite, 6-thioguanine, increasing the risk of myelosuppression.,56

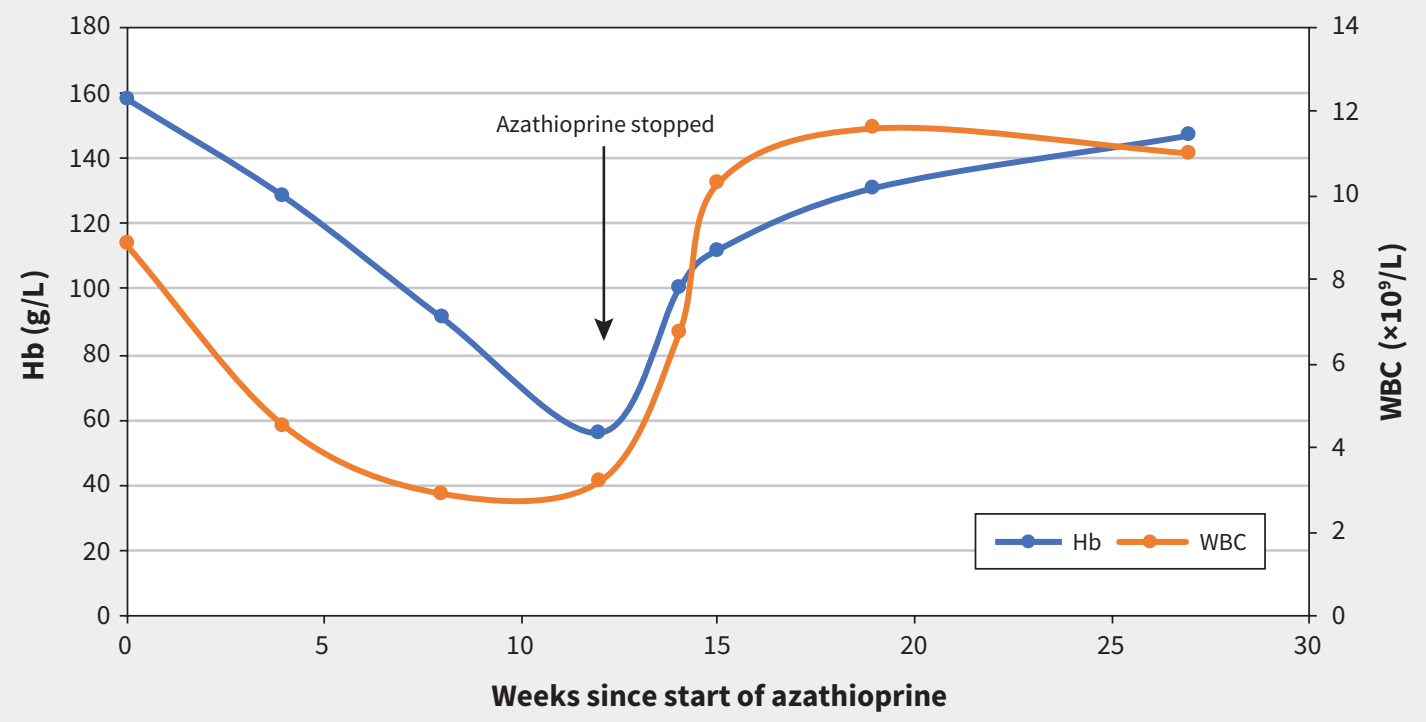

Figure 1: Hemoglobin $(\mathrm{Hb})$ and white blood cell $(\mathrm{WBC})$ count of a 66-year-old man taking azathioprine. Trend shows a gradual decline in $\mathrm{Hb}$ and WBC until the patient was admitted to hospital and azathioprine was stopped at week 12, after which $\mathrm{Hb}$ and WBC begin to improve. 


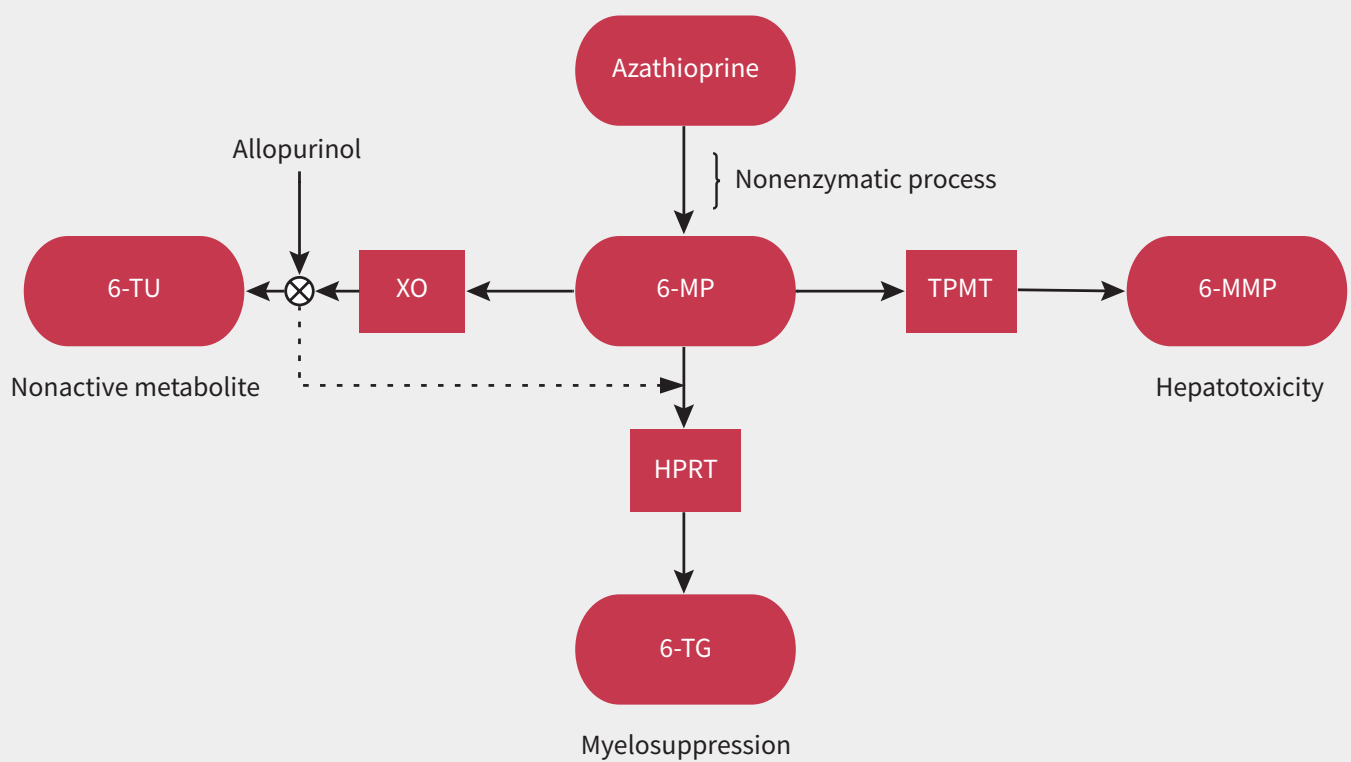

Figure 2: Pathways of azathioprine metabolism. Allopurinol inhibits the xanthine oxidase (XO) pathway, which normally metabolizes 6-mercaptopurine (6-MP) into 6-thiouracil (6-TU), a nonactive metabolite. Inhibition of the XO pathway leads to shunting toward the hypoxanthine phosphoribosyltransfirase (HPRT) pathway, increasing the production of 6-thioguanine (6-TG), thereby increasing the risk of myelosuppression..$^{2,3}$ Polymorphisms in the thiopurine methyltransferase (TPMT) enzyme can also cause shunting toward production of 6-TG. Hyperactivity in TPMT can increase the risk of hepatotoxicity through the production of 6-methylmercaptopurine (6-MMP).,3

About $11 \%$ of the general population has low TPMT activity, putting this group at risk of myelosuppression with the use of azathioprine. ${ }^{6}$ Medications such as 5-aminosalicylic acid, sulfasalazine and furosemide are also known to suppress TPMT activity and would similarly increase the risk of myelosuppression. ${ }^{6}$

The absence of a TPMT genetic mutation in our patient supports our hypothesis that an interaction between azathioprine and allopurinol, a xanthine oxidase inhibitor, was the main reason for his severe anemia. Moreover, both azathioprine and allopurinol modulate purine synthesis and have been implicated in megaloblastosis. ${ }^{9}$

These 2 medications have been safely combined in patients with inflammatory bowel disease, for whom combination therapy has been shown to help minimize the hepatotoxic effects of the 6-methylmercaptopurine metabolic pathway. ${ }^{7}$ However, when using azathioprine with allopurinol, a lower dose of azathioprine (about 25\%-50\% of the monotherapy dose), with weekly complete blood counts in the first month to monitor for toxic adverse effects, is suggested. ${ }^{5,7}$ The use of febuxostat, another xanthine oxidase inhibitor used as urate-lowering therapy for gout, should prompt similar precautions and dosing considerations. ${ }^{10}$ Other noteworthy drug-drug interactions with azathioprine and their mechanisms are listed in Table 1.1,4,6

\section{Time course of azathioprine toxicity and recovery}

According to case reports, severe anemia related to azathioprine develops in most patients within the first 2 months of drug

treatment. ${ }^{8,11}$ Similar to our patient, anemia tends to improve within 2 to 3 weeks after azathioprine is stopped. ${ }^{8}$

Stopping the use of azathioprine and suspected interacting drugs is the most important aspect of clinical management. Subsequent management of symptomatic anemia is supportive, consisting largely of blood product transfusions until the hemoglobin has stabilized. ${ }^{8}$

Table 1: Notable drug-drug interactions with azathioprine $e^{1,4,10}$

\section{Drug class}

Aminosalicylates

- Sulfasalazine

- 5-aminosalicylic acid

Angiotensin-converting-enzyme inhibitors

Warfarin

Xanthine oxidase inhibitors

- Allopurinol

- Febuxostat

\section{Drug effect or interaction}

Inhibits thiopurine methyltransferase pathway of azathioprine metabolism

Reported to induce anemia and leukopenia when used concurrently with azathioprine among patients who have had a renal transplant

Azathioprine may inhibit the anticoagulant effect of warfarin Inhibits the xanthine oxidase pathway of azathioprine metabolism 


\section{Monitoring and TPMT testing}

When starting azathioprine, a complete blood count with differential and liver enzymes should be monitored at least every 2 weeks during initial dose titration and, once stable, at least every 3 months thereafter, as clinically appropriate. ${ }^{12}$ Monitoring for and early detection of potential drug toxicity would guide interventions that might prevent adverse effects similar to what our patient experienced. Genetic testing for TPMT mutations should be considered in certain clinical settings. For example, the Canadian Gastroenterology Guideline recommends TPMT testing for patients with inflammatory bowel disease who are starting azathioprine to help guide dosage and identify patients at risk for severe toxicity. ${ }^{13}$

\section{References}

1. Gossmann J, Thürmann P, Bachmann T, et al. Mechanism of angiotensin converting enzyme inhibitor-related anemia in renal transplant recipients. Kidney Int 1996;50:973-8.

2. Meyboom RH, Hekster YA, Egberts AC, et al. Causal or casual? The role of causality assessment in pharmacovigilance. Drug Saf 1997;17:374-89.

3. Broen JCA, van Laar JM. Mycophenolate mofetil, azathioprine and tacrolimus: mechanisms in rheumatology. Nat Rev Rheumatol 2020;16:167-78.

4. Page RL II, Miller GG, Lindenfeld J. Drug therapy in the heart transplant recipient: part IV - Drug-drug interactions. Circulation 2005;111:230-9.

5. Sparrow MP, Hande SA, Friedman S, et al. Allopurinol safely and effectively optimizes tioguanine metabolites in inflammatory bowel disease patients not responding to azathioprine and mercaptopurine. Aliment Pharmacol Ther 2005;22:441-6.

6. Seidman EG. Clinical use and practical application of TPMT enzyme and 6-mercaptopurine metabolite monitoring in IBD. Rev Gastroenterol Disord 2003;3:S30-8.

7. Hoentjen F, Seinen ML, Hanauer SB, et al. Safety and effectiveness of longterm allopurinol-thiopurine maintenance treatment in inflammatory bowel disease. Inflamm Bowel Dis 2013;19:363-9.

8. Agrawal A, Parrott NR, Riad HN, et al. Azathioprine-induced pure red cell aplasia: case report and review. Transplant Proc 2004;36:2689-91.
9. Hesdorffer CS, Longo DL. Drug-induced megaloblastic anemia. N Engl J Med 2015;373:1649-58.

10. Jordan A, Gresser U. Side effects and interactions of the xanthine oxidase inhibitor febuxostat. Pharmaceuticals (Basel) 2018;11:51.

11. Alhubaishi AA. Pancytopenia and septic infection caused by concurrent use of allopurinol and mercaptopurine : a case report illustrating the importance of clinical pharmacist consultation. Am J Case Rep 2019;20:1245-7.

12. Warner B, Johnston E, Arenas-Hernandez M, et al. A practical guide to thiopurine prescribing and monitoring in IBD. Frontline Gastroenterol 2018;9:10-5.

13. Panaccione R, Steinhart AH, Bressler B, et al. Canadian Association of Gastroenterology Clinical Practice guideline for the management of luminal Crohn's disease. J Can Assoc Gastroenterol 2019;2:e1-34.

\section{Competing interests: None declared.}

This article has been peer reviewed.

The authors have obtained patient consent.

Affiliations: Department of Medicine (Madrazo, Jones) and Division of Hematology (Hsia), Department of Medicine, London Health Sciences Centre, University of Western Ontario, London, Ont.

Contributors: All authors contributed to the conception, drafting and revision of the manuscript. All authors contributed to the collection, analysis and interpretation of data included. All authors give final approval of the version to be published and agreed to be accountable for all aspects of the work.

Content licence: This is an Open Access article distributed in accordance with the terms of the Creative Commons Attribution (CC BY-NC-ND 4.0) licence, which permits use, distribution and reproduction in any medium, provided that the original publication is properly cited, the use is noncommercial (i.e., research or educational use), and no modifications or adaptations are made. See: https://creativecommons.org/licenses/by-nc-nd/4.0/

Correspondence to: Lorenzo Madrazo, Imadrazo@uwo.ca 